\title{
II metodo AEM, un nuovo strumento per l'idrogeologia
}

\author{
Antonio Menghini - Aarhus Geophysics ApS - Denmark \\ am@aarhusgeo.com
}

Andrea Viezzoli - Aarhus Geophysics ApS - Denmark

I progressi della Geofisica Applicata all'Idrogeologia sono stati talmente incisivi e rapidi che, oramai da diversi anni nei congressi internazionali, accanto alle sessioni più tradizionali, compare sempre più spesso un nuovo campo di applicazione: l'Idrogeofisica. La notevole crescita scientifico-tecnologica è stata indubbiamente favorita dalla marcata innovazione strumentale ed ha permesso di migliorare la qualità dei dati acquisiti, a favore sia della risoluzione superficiale che di quella in profondità. Parallelamente si è verificato un incisivo progresso nelle modalità di elaborazione ed inversione dei dati, anche sfruttando codici di joint-inversion o di utilizzo integrato di informazioni apriori derivanti da dati idrostratigrafici da pozzo o da più metodologie geofisiche. I metodi elettrici ed elettromagnetici, che sono in grado di descrivere la distribuzione della resistività (ovvero della conducibilità), un parametro strettamente correlato alla permeabilità degli acquiferi ed alla mineralizzazione delle acque sotterranee, sono quelli tra i più utili ai fini idrogeologici. Altra metodologia utile è la Risonanza Magnetica Nucleare (NMR), unica capace di rilevare direttamente la presenza d'acqua nel sottosuolo, oltre a fornire una stima dei parametri idrogeologici (trasmissività, porosità).

Il metodo Airborne EM rientra tra le metodologie EM più utili all'idrogeofisica, in quanto è in grado di fornire una distribuzione tridimensionale di estremo dettaglio della resistività, in tempi e con costi inimmaginabili se confrontati con le prospezioni realizzate a terra. Questa tecnica ha conosciuto negli ultimi anni un rapido sviluppo, che non accenna minimamente ad esaurirsi, tanto che, attualmente, sono disponibili sul mercato sistemi elitrasportati idonei a mappare accuratamente strutture anche molto superficiali, mentre altri aerotrasportati sono in grado di investigare sino a 500$600 \mathrm{~m}$ di profondità. Ovviamente la corretta elaborazione ed inversione del dato grezzo è un requisito fondamentale per un'applicazione di tipo idrogeologico, in quanto si hanno contrasti di resistività talvolta molto deboli, decisamente inferiori a quelli tipici della ricerca mineraria. Inoltre, è richiesta anche una elevata modellizzazione delle strutture (in termini di spessori, profondità, etc.), al fine di pianificare nel miglior modo possibile lo sfruttamento di nuove risorse idriche. I notevoli progressi nel campo dell'acquisizione e dell'inversione dei dati, permette di estrarre informazioni di notevole dettaglio, con la stessa risoluzione di una tomografia elettrica o di un sondaggio elettrico verticale. Possiamo pertanto asserire che si va dal "microscopico" al "macroscopico", con il vantaggio di avere un dataset congruo e omogeneo.

Indubbiamente uno dei punti di forza del metodo risiede nella capacità di acquisire dati con una notevole densità (di norma si estrae una maglia di 20x100 m), tale da rendere quanto mai affidabili i modelli idrogeofisici che possono essere ricostruiti, interpretando il dato geofisico insieme al contributo degli idrogeologi. L'area tipicamente indagata da una serie di prospezioni AEM è dello stesso ordine di grandezza di un bacino idrogeologico, quindi i modelli idrogeofisici che ne derivano possono essere poi facilmente importati nei software di modellizzazione idrogeologica, tipo ModFlow, MikeShe e FeFlow. La Figura illustra un esempio di interpretazione idrogeologica ottenuta con la metodologia AEM, in un ambiente glaciale (Joergensen \& Sandersen, 2009).

Tale approccio basato su acquisizione di grandi quantità di dati geofisici aerei, e loro integrazione con quelli idrogeologici, è stato implementato in Danimarca nel corso degli ultimi 10 anni, ed è una delle concause per cui la Danimarca è spesso indicata come uno dei fari in Europa dal punto di vista della gestione del ciclo integrato delle acque. Tale status è giustificato anche dal fatto che, per esempio, l'acqua estratta dagli acquiferi glaciali (profondità inferiore a $200 \mathrm{~m}$ ) venga solo ossigenata prima di essere distribuita e bevuta. Ciò è stato reso possibile dalla modellizzazione della vulnerabilità degli acquiferi, ottenuta integrando i dati areali di geofisica aerea con quelli puntuali diretti.

Questo modello, o alcuni dei suoi blocchi fondamentali quali l'utilizzo di geofisica aerea, è stato già applicato in altri paesi e altre situazioni idrogeologiche. Esempi ne sono le praterie del Nord America, alcuni bacini dell'Australia, parti della Francia metropolitana e coloniale. Il Ministero dell'Ambiente Danese, nell'ambito del Progetto "Rethink Water" (http:// rethinkwater.dk/) promuove l'ulteriore internazionalizzazione di questo approccio per la modellizzazione idrogeologica di interi bacini in diverse aree del mondo.

Nel caso di "Retbink Water" è stato creato un network di oltre 50 aziende danesi che spaziano da istituti di ricerca a università, da compagnie geofisiche a ditte di perforazione, da società di ingegneria ad aziende che operano nel settore del trattamento delle acque, con la prospettiva di seguire passo dopo passo tutto il ciclo che ha come oggetto l'acqua. Un ruolo fondamentale è svolto dal Servizio Geologico Danese (GEUS), che tra l'altro si occupa della gestione dei dati, sia di tipo stratigrafico, che idrochimico e geofisico. E' stato pertanto costituito un database nazionale che viene continuamente aggiornato e accessibile a qualsiasi utente, sia esso il comune cittadino che un Ente Pubblico. Inutile dire che con questo approccio lungimirante, si può parlare realisticamente di vera e propria "Pianificazione" delle risorse idriche sotterranee, poichè il Governo Danese ha tutti gli strumenti per ubicare nuovi campi-pozzi, nuovi insediamenti industriali e produttivi, o attività caratterizzate da particolare impatto ambientale.

Vale la pena menzionare altri due progetti internazionali: 
NICA (http://hgg.au.dk/projects/nica/; Schamper et al. 2012) che ha come obiettivo lo studio dell'inquinamento da Nitrati, per mitigare l'impatto dell'uso dei fertilizzanti sulle risorse idriche, e HyGEM (http://hgg.au.dk/projects/hygem; Auken \& Christiansen, 2012) che è centrato sull'uso sinergico di informazioni geofisiche, idrogeologiche e idrochimiche per migliorare la modellizzazione idrogeologica.

I vantaggi nel mappare le risorse idriche su larga scala non sono solo di tipo quantitativo (dove e quanta acqua trovare), ma anche qualitativo, in quanto la resistività elettrica è fortemente influenzata dal contenuto salino delle acque, tant'è vero che una delle applicazioni più consuete riguarda l'individuazione dell'intrusione marina nelle falde costiere. Di recente, per esempio, al congresso IdroVulc2013, è stata presentata una proposta di applicazione del metodo AEM per lo studio della distribuzione dell'Arsenico nell'acquifero vulcanico CiminoVicano (Menghini \& Viezzoli, 2013). La concentrazione di tale elemento dipende dalla natura del substrato delle vulcaniti, dalla presenza delle faglie che alimentano il circuito idrotermale, dal chimismo degli acquiferi vulcanici e dalla loro profondità; si tratta di caratteristiche che possono essere agevolmente verificate tramite una rigorosa ricostruzione ad alta risoluzione delle resistività nel sottosuolo, per cui si potrebbe realizzare una prospezione AEM, in grado di fornire in tempi ridotti (circa 3 mesi) un modello idrogeologico molto dettagliato (con maglia di circa 20x200 m, su di una superficie di circa $\left.25 \times 35 \mathrm{Km}^{2}\right)$.
Infine, numerosi sono i problemi di carattere idrogeologico che possono essere risolti tramite questo approccio multidisciplinare: si va dallo studio dell'intrusione salina lungo le falde costiere, ai fenomeni di subsidenza legati al sovrasfruttamento delle falde più superficiali o a processi di dissoluzione ed erosione di strati sepolti, dallo studio qualitativo degli acquiferi (fenomeni di inquinamento) alla localizzazione di campi-pozzi più produttivi, sino alla ricaptazione e recupero di vecchie opere di captazione e alla definizione delle aree di salvaguardia delle opere di captazione destinate al consumo umano.

\section{BIBLIOGRAFIA}

Auken E. \& Christiansen A.V. (2012) - Integrating Geophysics, Geology, and Hydrology to enhanced Hydrogeological Modelling. AGU, December 2012.

Joergensen F. \& Sandersen P.B.E. (2009) - 3D electromagnetic groundwater mapping - new contributions to the glacial geology in Denmark. Glaciogenic reservoirs and hydrocarbon system, Londra, 1-2 Dicembre 2009.

Klee P. (Ed.) (2013) - Greater water security with groundwater - Groundwater mapping and sustainable groundwater management. The Rethink Water network and Danish Water Forum white papers. www.rethinkwater.dk

Menghini A. \& Viezzoli A. (2013) - Proposta di applicazione del metodo AEM per migliorare le conoscenze idrostratigrafiche dell'acquiefro vulcanico Cimino-Vicano, con particolare riferimento al problema dell'Arsenico. Idrovulc2013, Orvieto, 16-17 Maggio 2013.

Schamper C., Auken E., Sørensen K.I. (2012) - Coil response inversion for very early time modeling of helicopter-borne time-domain EM data and mapping of near-surface geological layers; submitted to Geophysical Prospecting.
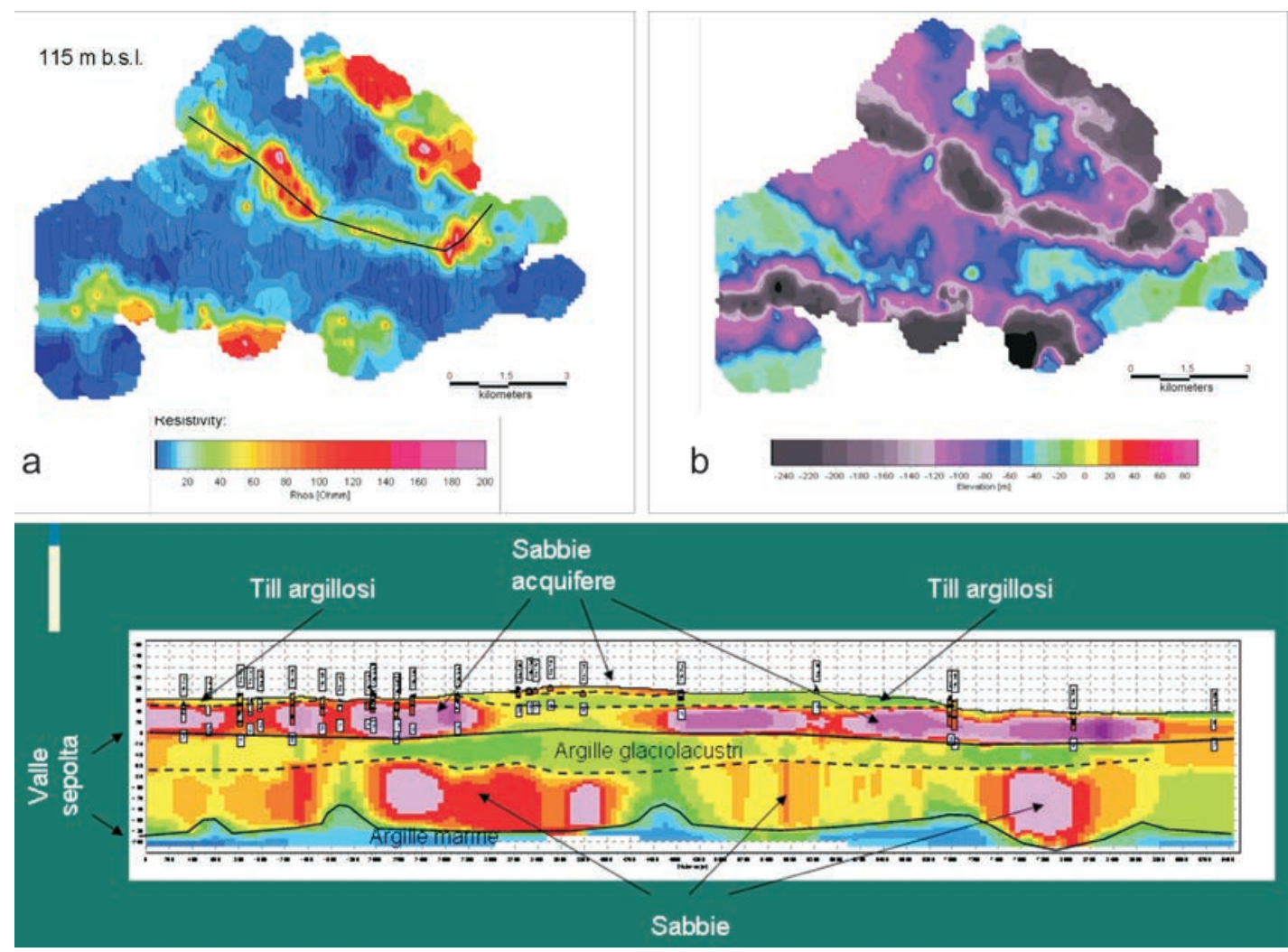

Fig.1: Esempio di risultati conseguibili con una prospezione AEM: a) mappa di resistività a 115 m di profondità; i puntini neri indicano $i$ sondaggi utilizzati per l'inversione; b) carta del tetto del substrato argilloso impermeabile (quote s.l.m.); c) sezione di resistività (traccia ubicata in a) con relativa interpretazione idrogeologica (rielaborato da Joergensen E Sandersen, 2009). 